\title{
Simplified model for calculation of clutters caused by wind turbines
}

\author{
Zdzisław Dziewit, Bogdan Bucki \\ Institute of Meteorology and Water Management - National Research Institute, Podleśna 61, 01-673 Warsaw, Poland, \\ e-mail: zdzislaw.dziewit@imgw.pl,bogdan.bucki@imgw.pl
}

\begin{abstract}
Several methodological attempts were made to assess the potential impact of a planned wind turbines on the operational work of weather radar. International recommendations for co-location have been established and incorporated in many countries. The main point of these recommendations states that the location of a wind turbine any part of which reaches RLOS (Radar Line Of Sight) requires the analysis of its impact on radar performance. The growing number of applications for the technical assessment of planned wind farms demand the use of a simple method of calculation for the first step of the authorization process.

A typical wind turbine consists of several metallic parts. Based on the assumption that only moving, metallic parts of a turbine cause echoes indelible by standard filtering used in radar signal preprocessing, a method of preliminary evaluation of such maximum potential clutters was developed. The method takes into account how great a part of a turbine is really illuminated by a radar beam and for this part calculates a RCS (Radar Cross Section) and its equivalent radar reflectivity. A very detailed description of the model is given in this article in order for it to be easy to implement in any calculation system. Discussion of the influence of the main simplifications assumed in this model, as well as a comparison of the theoretical results with example data in operational mode of radar work, are included in this article.
\end{abstract}

Keywords: clutter model, weather radar and wind turbine co-existence, RCS, equivalent radar reflectivity, wind farm planning

Submitted 13 October 2015, revised 25 October 2016, accepted 24 January 2017

\section{Introduction}

The need for renewable energy is increasing all over the World. This leads to the need for the installation of new wind farms. The first diagnostic studies of the influence of wind turbines on radar observations began more than 20 years ago (DoD 2006). Since that time a lot of experience has been gained, recommendations for co-location have been established (OPERA 2010; WMO 2010; Met Office 2012; NOAA 2015; etc.) and formal procedures of the investment process have been incorporated in many countries. The main point in the recommendations states that the location of a wind turbine of which any part reaches RLOS (Radar Line Of Sight) needs analysis of its impact on radar performance.

Several methodological attempts have been made in recent years to assess the potential impact of planned wind turbines on the operational work of weather radar. The approaches vary significantly, starting from case-by-case, very detailed modeling of the influence using dedicated software and very exact modeling of the environment (shape of land, buildings, vegetation etc.), the turbine itself (shape and materials), weather radar components (antenna, electronic parts, signal processing), and their relative movement etc. (Bobillot et al. 2012) At the other end there are very simplified models based on theoretical simulations of RCS (Radar Cross Section) of a typical, idealised (and simplified) turbine model, converted into radar reflectivity with the assumption that the whole wind turbine is included within the radar beam (Angulo et al. 2015). The first approach requires a lot of effort and powerful computers to reach its results and has to be performed when the coexistence of wind farm and weather radar is unavoidable, and we also have to be aware of the detailed impact on different radar products. While the second approach, as it is intended to be used in the very first stage of finding potential places for turbine location, doesn't deal with true turbine details, its results could still be useful.

A typical wind turbine consists of several metallic parts. Some of them are relatively big but generally they are non-moving, e.g. tower, nacelle. Some other parts are small but moving, e.g. lightning rods in turbine blades. Echoes coming from non-moving (or very slowly moving) objects, even with relatively high reflectivity, are quite successfully filtered by the data processors of modern radars, or can be made "stealth" by special treatment of their surface (Cameron 2011). Moving objects are deemed by a radar processor to be a "weather object" and contaminate a received signal. In the case of the typical rotor of a modern wind turbine, the main part of the blade reflects 
a low amount of energy because of the material it is made of (Glass Reinforced Plastic) ${ }^{1}$. Unfortunately, lightning rods protecting a turbine have to be made of metal and its ability to reflect radar radiation is very high. The lightning rod forms a long (when compared to radar wave length), thin conductor. Recently a great effort has been made to work out "stealth blades" (Cameron 2011). Regardless of the significant reduction of RCS of blades, lightning protection systems still cause great reflectivity (Danoon et al. 2014). Up to now, no Polish investor applying to IMWM has declared that they want to use such technology. Because of the reported very high variability of RCS (Danoon et al. 2014), such investment shall be treated uniquely and is not considered in our model.

The Polish Institute of Meteorology and Water Management (IMWM) is a stakeholder in the authorisation process of installing new wind turbines on Polish territory in the vicinity of radar stations. For optimal use of its limited resources, IMWM had to simplify the decision process in its first stages. A tool was required which was simple in use, but realistic. Based on the assumption that only moving, metallic parts of a turbine cause echoes indelible by standard filtering, a method of preliminary evaluation of such clutter was developed. The method takes into account how large a part of a turbine could really be illuminated by a radar beam.

We are aware that reflection from wind turbine is extremely variable in time and effective reflectivity quite rarely reaches its maximum possible value. Our target was not to make a tool for real time estimation of true temporary reflectivity, but to make a tool for easy estimation of maximum possible effective reflectivity (not taking into account some additional effects and temporary layout configurations). Our analysis shown in the last section serves as a conclusion that such additional effects are smaller than reflection from lightning rods.

\section{Proposed simplified model for calculation of clut- ters caused by a wind turbine}

The simplified model of a wind turbine considered in this article is based on the following assumptions:

- only the reflectivity aspect of spurious echo is considered;

- non-moving (or very slowly moving) parts of a wind turbine can be neglected because of the quite successful Doppler filtering by modern radar processors;

\footnotetext{
${ }^{1}$ Theoretically, problems may appear when the surfaces of the blades become wet due to rain (large conducting surface), but our experience shows that this is not the case. More details will be presented in further publications.
}

- the only parts which cannot be filtered out are metallic lightning rods in turbine blades (long, thin electric cables);

- the turbine rotor consists of three blades ${ }^{2}$;

- we always take "the worst case", i.e., such an orientation of blades and beam which gives the highest reflections ("blade flashes");

- radar is a typical modern pulse weather radar $^{3}$, scanning with a narrow beam rotated azimuthally, no dedicated signal filtering is applied (except for standard Doppler filter);

- there are no obstacles between radar and the turbine shadowing the radar beam.

The description of the model given below takes in elements known in one place, and complements this with intermediate equations and detailed specifications to make it easy to implement in any calculation system.

\subsection{Only reflectivity aspect of spurious echo}

Of course not only reflectivity data are contaminated by wind turbines; Doppler data and dual-pol data are contaminated as well. The proposed model was constructed for use in the evaluation of possible disturbances by a planned turbine. The first approach is to assess the level of an irremovable reflectivity signal. From our experience it can be stated that cases when deeper analysis has to be done are very rare up to now in Poland. Typically a location can be accepted, or has to be rejected. So, due to the increasing number of submitted applications for assessment, it is a good option to have a simple tool to easily perform the first approach.

\subsection{The only parts which cannot be filtered out are me- tallic lightning rods in turbine blades (long, thin electric cables)}

Although it is possible that not only lightning rods in moving parts of a turbine are made of metal, the lightning rods are indispensable in the construction of such tall objects. Therefore, lightning rods could be treated as the first approach in calculations. If the first approach results in low enough reflectivity, a more detailed examination has to be performed. But, from our experience, such cases are rather rare. For example, according to our model, a wind turbine located $20 \mathrm{~km}$ from a radar and exhibiting for

\footnotetext{
${ }^{2}$ It is a very typical construction. In the proposed model the total illuminated length of lightning rods is evaluated. The real number of turbine blades could cause some error - see the chapter with the discussion on some aspects of the proposed model.

${ }^{3}$ Further in this article, the example values of calculated or measured parameters are presented for $5.65 \mathrm{GHz}$ of operating frequency.
} 
a radar beam, a lightning rod of diameter $13.8 \mathrm{~mm}$ with only $1 \mathrm{~m}$ length gives $26.5 \mathrm{dBZ}$ of irremovable reflectivity, but with length of $10 \mathrm{~m}$ it gives $46.3 \mathrm{dBZ}$ [29 mm/h), which is a result that cannot be accepted. The same $1 \mathrm{~m}$ lightning rod at a distance of $100 \mathrm{~km}$ would give $10.5 \mathrm{dBZ}$.

\subsection{The shielding effects of the composite materials of the blades}

Modern wind turbine blades are made of composite materials. The materials envelop the lightning rod, causing some shielding against radar microwave radiation. The method and materials for producing the blades is almost always a trade secret and is not known publicly. Additionally, an investor doesn't decide about a turbine's manufacturer and model at a very early stage in the process of locating turbines. It is therefore impossible to take this effect into consideration. Some guess could be made by comparison with radar radome which is also made of composite material. The disadvantage of this guess is that materials of radome are carefully selected to minimise microwave attenuation. Regardless of this, typical oneway attenuation in radome is of the order of $0,2 \mathrm{~dB}(\mathrm{CSZH}$ 2014), so one can expect that the shielding has a minor influence on the final clutter estimation. It must be emphasised that the proposed model estimates "the worst case", i.e., the most possible contamination.

\section{Scheme of calculations of spurious echo}

The theoretical calculation of the reflectivity of the abovementioned lightning rod can be carried out in the following steps:

1) Calculate the power density of microwave radiation approaching the wind turbine from radar.

2) Calculate the length of wire (lightning rod) illuminated by radar radiation.

3) Calculate the radar cross-section of the illuminated part of wire.

4) Calculate the power coming back to radar as back-scattered by the wire.

5) Recalculate it into the equivalent radar reflectivity. The above scheme of calculations was worked out in IMWM to fulfill its obligations in the authorisation process of the installation of new wind turbines.

\subsection{Power density of microwave radiation approach- ing a wind turbine from a radar}

Calculation of the power density may be carried out with following equation:

$$
W_{i, \text { corrected }}=(10)^{\left(\frac{W_{i, d B, \text { corrected }}}{10}\right)}
$$

where: $W_{i, \text { corrected }}$ - power density in linear scale $\left[\mathrm{W} \mathrm{m}^{-2}\right]$; $W_{i, d B, \text { corrected }}$ - power density in logarithmic scale $[\mathrm{dB}]$;

$$
W_{i, d B, \text { corrected }}=10 \cdot \log _{10}\left(W_{i}\right)-L_{t x}-L_{\text {radome }}-\frac{c_{a t h m}}{2} \cdot d
$$

where: $W_{i}$ - power density calculated without any losses on the way between transmitter and wind turbine $\left[\mathrm{W} \mathrm{m}^{-2}\right]$; $L_{t x}-$ losses in waveguide between transmitter and antenna $[\mathrm{dB}] ; L_{\text {radome }}$ - one-way losses in radome $[\mathrm{dB}] ; c_{\text {athm }}-$ twoway losses in atmosphere $\left[\mathrm{dB} \mathrm{km}^{-1}\right] ; d$-distance between radar and turbine $[\mathrm{km}]$.

Values of the losses are taken as results of measurements made during regular radar maintenance procedures (SELEX ES 2015).

$$
W_{i}=\frac{P_{0} \cdot G}{4 \cdot \pi \cdot(d \cdot 1000)^{2}}
$$

where: $P_{0}$ - power of microwave pulse generated by radar transmitter [W]; $G$ - linear antenna gain $\left[\mathrm{WW}^{-1}\right]$.

$$
G=(10)^{\left(\frac{G_{d B}}{10}\right)}
$$

where: $G_{d B}$ - nominal antenna gain in decibels measured during maintenance procedure $[\mathrm{dBi}]$.

The important parameter in equations (2) and (3) is distance $d$ between radar and turbine. This parameter can be calculated with the following equation (Meeus 1999):

$d=R_{z} \cdot \arccos \left(\sin \varphi_{W T} \cdot \sin \varphi_{r}+\cos \varphi_{W T} \cdot \cos \varphi_{r} \cdot \cos \left(\lambda_{W T}-\lambda_{r}\right)\right)(5)$

where: $R_{z}$ - average radius of the Earth, $6371 \mathrm{~km} ; \varphi$ - latitude $\mathrm{N}$ (WGS84) $\left[{ }^{\circ}\right] ; \lambda_{(\ldots)}-$ longitude $\mathrm{E}$ (WGS84) $\left[{ }^{\circ}\right]$ and the references mean: $W T$ for wind turbine and $r$ for radar. Result of $\operatorname{arc} \cos ()$ has to be in radians. We assume a round Earth, which provides good accuracy for our needs.

\subsection{Length of wire (lightning rod) illuminated by ra- dar radiation}

Calculation of length $L$ of wire (lightning rod) illuminated by radar radiation - has to be carried out with geometrical considerations, taking into account: distance between radar and turbine, curvature of the Earth and the microwave propagation path in the atmosphere, relative heights of radar and turbine, as well as possible obstacles between radar and turbine ${ }^{4}$.

The considered geometry is shown on Fig. 1.

\footnotetext{
${ }^{4}$ Obstacles are not considered in this article.
} 


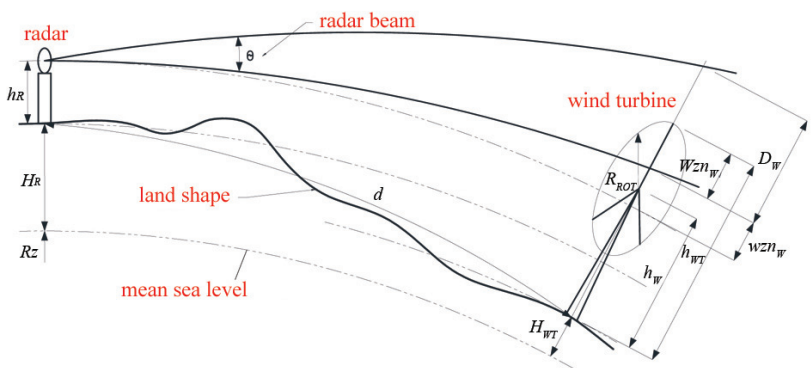

Fig. 1. Geometry of radar - wind turbine interaction

At first we have to calculate how large a part of a turbine rotor is illuminated by a radar beam. To do so we need to calculate the difference between the top of a rotor blade (pointed vertically) and the edges of a radar beam ( $W z n_{w}$ for lower edge). We assume that the beam has angular width $\Theta_{\text {rad }}$ and has a constant characteristic inside (and zeroed outside). No side lobe is taken into account.

The rotor blade is not parallel to the turbine tower in the real wind turbines, but is slightly slanted in order to avoid the blade, curved by the wind, hitting the mast. As a result, the effective length of a lightning rod (as seen from radar) is a little bit smaller, but even for a $5^{\circ}$ slant the difference is in the order of $0,4 \%$ and we neglect the effect.

Let's go through the following auxiliary equations.

For the calculation of the elevation of the lower beam edge over the level of antenna centre, but at the location of a wind turbine (i.e., at distance $d$ from radar), simple geometrical considerations lead us to the equation:

$$
w z n_{W}=R_{e} \cdot\left(\frac{1}{\cos \alpha-\sin \alpha \tan \beta}-1\right) \cdot 1000
$$

where: $w z n_{w}$ - elevation of lower beam edge over the level of antenna centre, but at the location of a wind turbine (i.e., at distance $d$ from radar) [m]; $R_{e}$ - effective radius of the Earth $=8493 \mathrm{~km}$ (equal to $4 / 3$ of the Earth's real radius) (Doviak, Zrnič 2006); by using this we take into account standard refraction in the atmosphere; $\alpha=\frac{d}{R_{e}}-$ auxiliary quantity (the angle from the Earth's centre, between radar and turbine) [rad]; $\beta=\beta_{n}-\frac{\theta_{\text {nad }}}{2}-$ auxiliary quantity, where $\beta_{n}$ is the elevation angle of radar antenna tilt, taken for consideration; typically equal to $0.5^{\circ}$ [rad].

According to Fig. 1, the difference between the top of a rotor blade (pointed vertically) and the edges of a radar beam $\left(W z n_{w}\right)[\mathrm{m}]$ is equal to:

$$
W z n_{w}=H_{R}+h_{R}+w z n_{w}-H_{W T}-h_{W T}
$$

where: $H_{R}$ - elevation (above mean sea level) of the base of radar tower [mAMSL]; $h_{R}$ - height (above ground level) of the centre of an antenna [mAGL]; $H_{W T}$ - elevation (above mean sea level) of the base of a wind turbine tower [m AMSL]; $h_{W T}$ - height (above ground level) of the top part of a wind turbine, i.e., the top of a rotor blade (pointed vertically) [mAGL].

A negative value of $W z n_{w}$ means that interaction occurs between a radar beam and the rotor of a wind turbine.

Relying on parameter $W z n_{w}$, we can enumerate five cases of how a rotor is located against a radar beam (Fig. 2) ${ }^{5}$ :

1) the whole rotor is under the beam cross section;

2) the upper section of the rotor intersects the lower part of the cross section of the beam;

3) the whole rotor is inside the beam cross section;

4) the lower section of the rotor intersects the upper part of the cross section of the beam;

5) the whole rotor is above the beam cross section.

We assume that a wind turbine is far enough from a radar so that the beam diameter is greater than the rotor diameter. In other cases the next calculations can lead to inaccuracies in calculated values. In practice, i.e., for a rotor diameter of about $90 \mathrm{~m}$, the distance between a radar and turbine shall be not less than $5 \mathrm{~km}$.

The first case gives a perfect location of a wind turbine because it will not influence a radar (turbine is out of RLOS). The second case is "hopeful". The intersection could be small enough to accept the location because of the
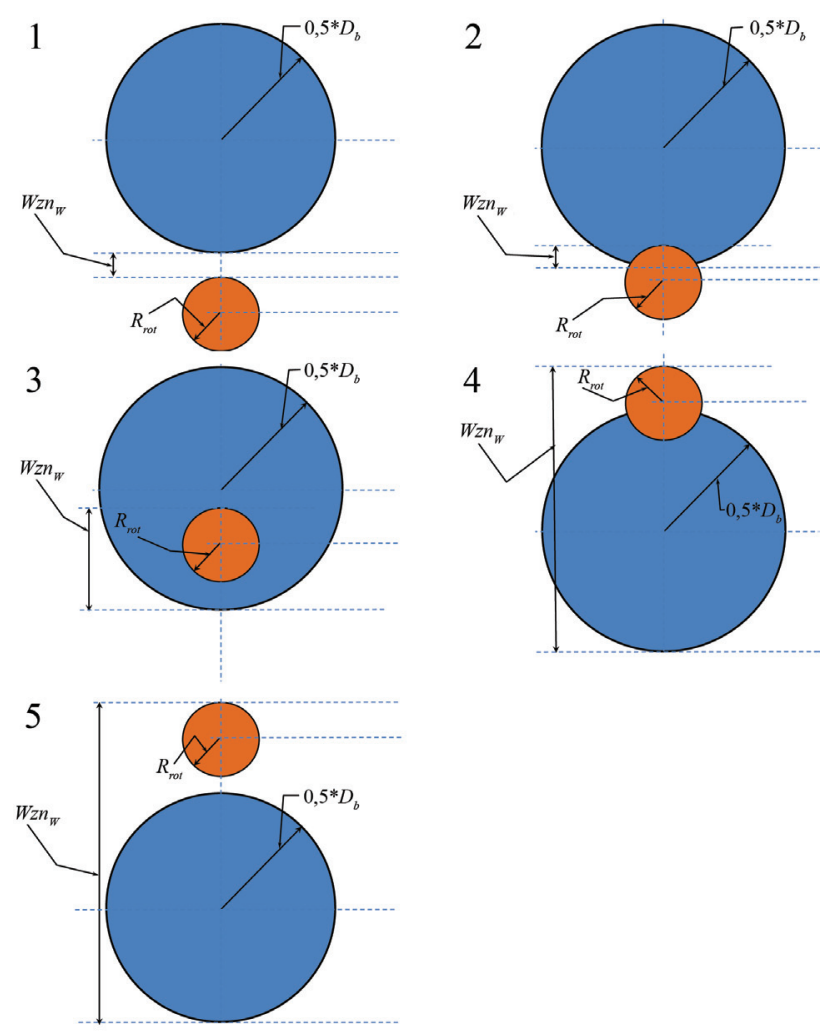

Fig. 2. Relative location of radar beam (with diameter $D_{b}$ ) and turbine rotor (with radius $R_{\text {rot}}$ ) as listed in the text above

\footnotetext{
${ }^{5}$ For simplicity we consider the rotor as a circle.
} 

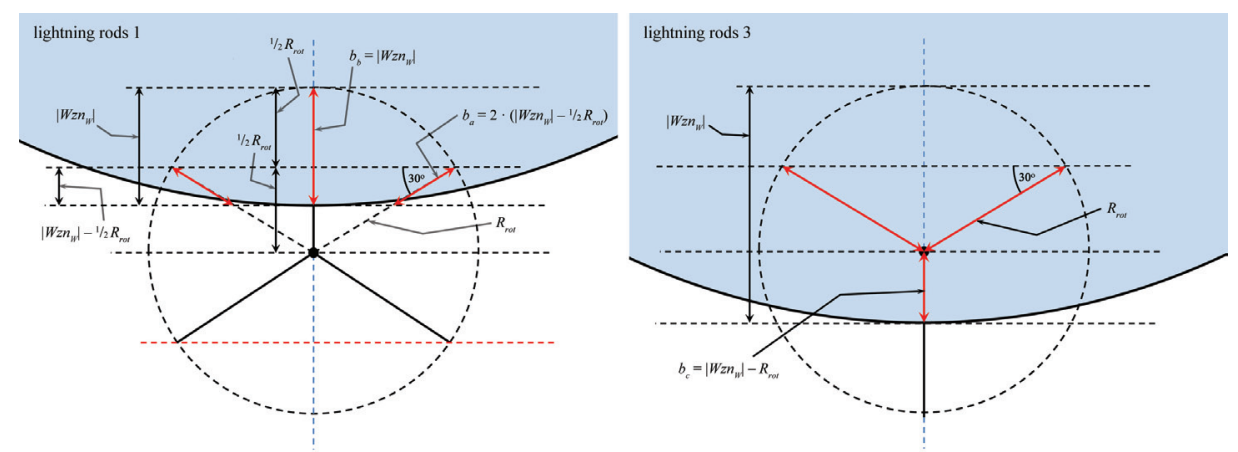

Fig. 3. Relative location of rotor blades and radar beam

low level of spurious echoes. The third case always gives large reflections, even at a large distance $d$. The fourth and fifth cases give similar effects as the third case because the rotor will be totally illuminated by the radar beam at the second (or next) elevation angle.

To finalise the second step of our calculations (finding the length of an illuminated wire) we shall consider which parts of the rotor blades (i.e., wires) intersect a radar beam. Figure 3 will be useful.

To simplify our calculations we will find the total length $L$ of the illuminated parts of wire. The nonlinearity between the length of wire and its radar cross section will lead to inaccuracies in reflectivity, but we will discuss later the possible magnitude of the inaccuracies. Another assumption is that beam diameter is significantly greater than rotor diameter (i.e., the edge of the beam circle, crossing the rotor circle, is almost "flat"). The longest total length of illuminated wire occurs when one of the blades is located vertically (up or down) and the next two blades are located at an angle $\pm 30^{\circ}$ to the horizon.

Four cases shall be considered (two of them shown in Fig. 3). Those illuminated are:

1) only one blade pointed vertically up;

2) only two blades pointed up at an angle of $+30^{\circ}$ to the horizon;

3) two blades pointed up at an angle $+30^{\circ}$ to the horizon and part of a blade pointed vertically down;

4) full length of all blades.

From geometrical considerations we can see that the illuminated length of wire $L_{a c t}$ and the limits separating the cases are as follow:

1) $L=b_{b}=\left|W z n_{w}\right|$ for $0<\left|W z n_{w}\right| \leq(2 / 3) \cdot R_{r o t}$

2) $L=2 \cdot b_{a}=4 \cdot\left|W z n_{W}\right|-2 \cdot R_{\text {rot }}$ for $(2 / 3) \cdot R_{\text {rot }}<\left|W z n_{w}\right| \leq R_{\text {rot }}$

3) $L=2 \cdot R_{r o t}+b_{c}=\left|W z n_{W}\right|+R_{\text {rot }}$ for $R_{r o t}<\left|W z n_{w}\right| \leq 2 \cdot R_{\text {rot }}$

4) $L=3 \cdot R_{\text {rot }}$ for $2 \cdot R_{\text {rot }}<\left|W z n_{w}\right|$

\subsection{Radar cross-section of the illuminated part of wire}

Calculation of a radar cross-section $\sigma_{\text {wire }}$ of the illuminated part of a wire can be made on the assumption that the length of wire is large and the radius is small compared to wave length. In such cases the following equation may be used (Crispin 2013):

$$
\sigma_{\text {wire }}=\pi \cdot L^{2} \cdot\left(\frac{\phi}{2 \cdot \lambda_{\text {wave }}}\right)^{0,57} \text { for: } \frac{L}{\lambda_{\text {wave }}}>1
$$

where: $\sigma_{\text {wire }}$ - radar cross section (in linear scale) of the illuminated part of wire $\left[\mathrm{m}^{2}\right] ; L-$ length of the illuminated part of wire $[\mathrm{m}] ; \phi-$ diameter of wire $[\mathrm{m}] ; \lambda_{\text {wave }}-$ wave length of radar radiation $[\mathrm{m}]$.

The above equation is valid with the assumption that lightning rod is parallel to polarisation of the radar wave. In cases where the rod is perpendicular, the backscattered signal can be up to 10-25 dB weaker. However, bearing in mind that we are looking for the "worst" possible clutter, we assume for following calculations that the rod is parallel.

\subsection{Power coming back to radar as back-scattered by the wire}

Calculation of power received by radar as back-scattered by the wire $P_{r, d B m}$, corrected - may be carried out in the following way.

First, calculate the density of power reflected from the wire, measured at radar location $W_{D}$ :

$$
W_{D}=\frac{\sigma_{\text {wire }} \cdot W_{i, \text { corrected }}}{4 \cdot \pi \cdot(d \cdot 1000)^{2}} \cdot 10^{3}
$$

where: $W_{D}$ - density of power (in linear scale) reflected from the wire, measured at radar location (i.e., after coming back to radar) $\left[\mathrm{mW} \mathrm{m}^{-2}\right] ; W_{i, \text { corrected }}-$ see equation (1).

Then, power received by radar expressed in logarithmic scale $P_{r, d B m}$ is equal to:

$$
P_{r, d B m}=10 \cdot \log _{10}\left(W_{D} \cdot A_{e}\right)
$$

where: $P_{r, d B m}$ - power (in logarithmic scale) reflected from the wire, received by radar (no correction up to now) $[\mathrm{dBm}] ; A_{e}-$ effective area of radar antenna $\left[\mathrm{m}^{2}\right]$. 
And finally, after necessary corrections:

$$
P_{r, d B m, \text { corrected }}=P_{r, d B m}-\frac{c_{a t h m}}{2} \cdot d-L_{r a d o m e}-L_{r x}
$$

where: $P_{r, d B m, \text { corrected }}$ - corrected power (in logarithmic scale) reflected from the wire, received by radar $[\mathrm{dBm}]$; $L_{r x}$ - losses between antenna and input point of radar processor $[\mathrm{dB}]$. Values of the losses are taken as the results of measurements made during regular radar maintenance procedures (SELEX ES 2015).

\subsection{Equivalent radar reflectivity}

Recalculation of power coming back to radar into equivalent radar reflectivity $Z$ [dBZ] shall be made with the weather radar equation (SELEX ES 2015):

$$
Z=C_{\text {radar }}+P_{r, d B m, \text { corrected }}+20 \cdot \log _{10}(d)
$$

where: $C_{\text {radar }}$ - radar constant, as measured during maintenance procedure (SELEX ES 2015).

\section{Discussion of some aspects of the proposed model 4.1. Neglecting the influence of non-moving parts}

The standard Doppler filter can act in various manners, but the result (removal of echoes from a non-moving object) may be characterised by depth of attenuation of signal components of around $0 \mathrm{~m} / \mathrm{s}$ (clutters) (SELEX ES 2015). Typically the attenuation values are in the range 30 $50 \mathrm{~dB}$. So, even quite strong clutters could be rejected.

Values for RCS calculated by detailed modeling of a typical wind turbine (Angulo et al. 2015) are in the scope of 49-68 dB. The residual signal after standard Doppler filtering depends on configurable filter attenuation and on the distance between radar and turbine. The residual signal is at an acceptable level in the sense that "detected rainfall" intensity doesn't cause alarm. Of course, long term total amounts of precipitation will show unrealistic values, but this may be treated in preprocessing data for climatic models. On the other hand, because of $\mathrm{CCOR}^{6}$ thresholding, true signals coming from real, weak precipitation would be removed.

\subsection{The turbine rotor consists of three blades and the blades are illuminated steadily}

There was a simplification made in the proposed model, in equation (8), by using the value of the total length

${ }^{6}$ The clutter correction ratio (CCOR) value is defined as the logarithmic ra-
tio between the unfiltered signal power and the clutter filtered signal power. of all illuminated parts of lightning rods as the length of illuminated wire. Because of the nonlinearity of $L$ in this equation, the maximum error occurs when the length consists of three parts. In such a case one can calculate that:

$$
3 \cdot \sigma_{\text {wire }}\left(\frac{L}{3}\right)=3 \cdot \pi \cdot\left(\frac{L}{3}\right)^{2} \cdot\left(\frac{\phi}{2 \cdot \lambda}\right)^{0,57}=\frac{1}{3} \cdot \sigma_{\text {wire }}(L)
$$

So: $\sigma_{\text {wire, calculated }}=3 \cdot \sigma_{\text {wire, real }}$, and in logarithmic scale: $\sigma_{\text {wire, }}$ real, $d B=\sigma_{\text {wire, } \text { calculated, } d B}-4.77 \mathrm{~dB}$.

We can say that the overestimating of the proposed model is not higher than $4.77 \mathrm{dBZ}$. And it should be noted that such a case (three parts of lightning rods are illuminated simultaneously) means that (almost) all blades are in a radar beam. Because modern blades are about $40 \mathrm{~m}$ in length, the total clutter is in the order of $67 \mathrm{dBZ}$. In such a case $-4.77 \mathrm{dBZ}$ doesn't change the verdict.

If more accurate values are necessary, a deeper analysis will have to be performed.

\subsection{Always take "the worst case" of blade orientation.}

In the case of a long wire $\left(L>>\lambda_{\text {wave }}\right)$, the most effective reflection is made in a direction perpendicular to the axis of this wire (i.e. axis of this elongated cylinder). For a three-blade rotor, the highest reflection occurs when the rotor axis is directed toward a radar. But on the other hand, in such a situation the radial velocity of all elements is equal to zero. So, we have to calculate at what value of angle between the rotor axis and the direction to radar the radial velocity is high enough to not be filtered by Doppler filter.

Let's consider the situation shown in Fig. 4.

From geometrical considerations we can say that radius $r$ at which the linear speed of a rotor blade reaches the value of radial velocity $V_{\text {radial }}$ is equal to:

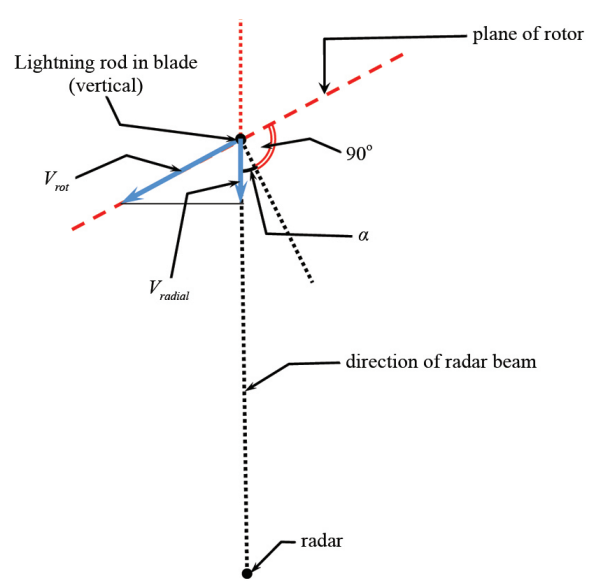

Fig. 4. Geometry of moving blade (with lightning rod inside) and its direction to radar 


$$
r=\frac{V_{\text {radial }} \cdot 60}{2 \cdot \pi \cdot f_{\text {rot }} \cdot \sin (\alpha)}
$$

where: $r$ - radius (distance from rotation axis to moving part) $[\mathrm{m}] ; f_{\text {rot }}$ - rotation speed [revolution minutes ${ }^{-1}$; $V_{\text {radial }}$ - linear speed towards radar $\left[\mathrm{m} \mathrm{s}^{-1}\right] ; \alpha$ - angle between perpendicular to rotor plane and direction to radar $\left[{ }^{\circ}\right]$.

Assuming that the radar is configured to remove signals from objects moving with velocities lower than $V_{\text {radial,filtered }}$, we come to the conclusion that the radar at least removes parts that fulfill the following condition:

$$
r \cdot \sin (\alpha)<\frac{V_{\text {radial, filtered }} \cdot 60}{2 \cdot \pi \cdot f_{\text {rot }}}
$$

where: $V_{\text {radial, filtered }}$ - maximum velocity of signals (objects) removed by Doppler filter $\left[\mathrm{m} \mathrm{s}^{-1}\right]$.

Let's look at an example: length of blade equal to $45 \mathrm{~m}$, rotor rotation speed $20 \mathrm{rev} / \mathrm{min}$., Doppler filter index 9 (absolute width of filter $1,5 \mathrm{~m} / \mathrm{s}, V_{\text {radial, filtered }}=0,75 \mathrm{~m} / \mathrm{s}$ ), wave length of radar radiation $5,3 \mathrm{~cm}$, pulse repetition frequency (PRF) $1200 \mathrm{~Hz}$. The maximum, filtered length of part of rotor as function of angle $\alpha$ is shown in Fig. 5.

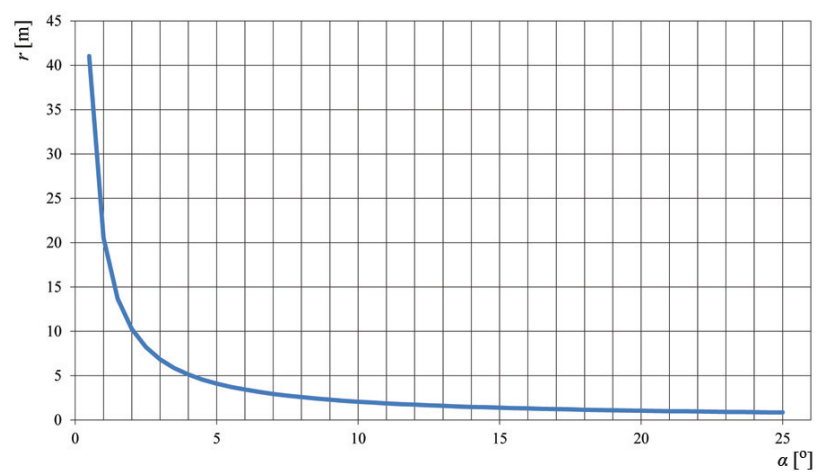

Fig. 5. Distance from rotor axis to end of filtered-out part of moving blades (for example parameters as described in the text)

It is easily seen that only for very small values of angle $a\left(<5^{\circ}\right)$ will the Doppler filter remove a significant part of the echoes coming from rotating blades. So, this could affect only a very small part of radar operational time.

\section{Real example - data in operational mode of radar work}

Bearing in mind all assumptions made in this model and remembering that it has been argued that RCS is not applicable (because of the omitted aspects) to wind turbines (when detailed modeling) (Greving, Malkomes 2006), to have a first guess about the effectiveness of the presented simplified model and its applicability in real cases, we examined operational data from the radar site Legionowo, close to Warsaw (Poland), for the year 2013.
Five wind turbines Vestas V90 are located at a distance of 22 to $25 \mathrm{~km}$ from the radar (in a north-west direction) and form Dębinki wind farm. All turbines on the farm are located on a top of a hill and therefore the whole rotor is illuminated ${ }^{7}$ (it corresponds to the $3^{\text {rd }}$ part of Fig. 2). Detailed information and results calculated according to the proposed model are summarised in Table 1.

The theoretical maximum clutter calculated according to the model gives a value of $67.9 \mathrm{dBZ}$. The true reflectivity (i.e. measured during operational work) found on the PPI map, 21:00 UTC 6 June 2015, at the location of the farm, shows a value of $62.5 \mathrm{dBZ}$ (Fig. 6). The corresponding volume data (raw data in spherical coordinates before reprocessing into map) shows a value of $64 \mathrm{dBZ}$. The data are after Doppler filtering. No weather reflectivity or other source echoes were present at that time in the Dębinki wind farm location.

It is very difficult to evaluate the effect of Doppler filtering of the middle part of the turbine rotor. Data about wind direction at that time, taken from a numerical weather model (i.e. after assimilation of synoptic data), show that the direction pointed very well from Dębinki (wind farm) to Legionowo (radar station) with an accuracy of a dozen or so degrees, but we don't have true in situ measured data. In such a case the effect described in part 3 can lead to a decrease in reflectivity by a few $\mathrm{dBZ}$; for example, for $\alpha=2,5^{\circ}$ the reduction will be $-3,4 \mathrm{dBZ}$. It has to be remembered that in this case every small modification in direction of rotor axis can lead to large changes in reflectivity (see Fig. 5).

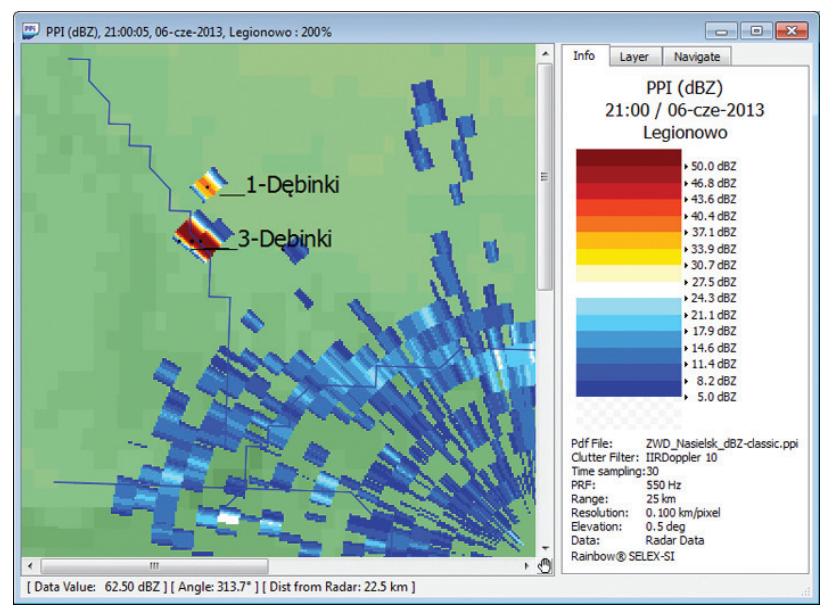

Fig. 6. An example of operational data showing the wind farm in Dębinki (Poland)

${ }^{7}$ According to the previously mentioned overestimation caused by using the aggregated length of wires as the length of the reflecting object in the calculations, the model output was corrected by $-4.77 \mathrm{dBZ}$, resulting in the value $67.9 \mathrm{dBZ}$ after correction. 
Table 1. Detailed information about Dębinki wind farm and results calculated according to the proposed model

\begin{tabular}{|c|c|c|c|c|c|c|c|c|c|c|c|c|c|}
\hline \multirow[t]{3}{*}{$\begin{array}{l}\text { İ } \\
\text { Zू }\end{array}$} & \multicolumn{3}{|c|}{ 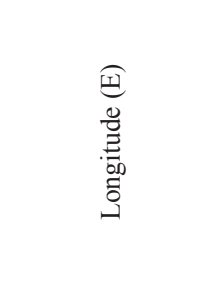 } & \multicolumn{3}{|c|}{ 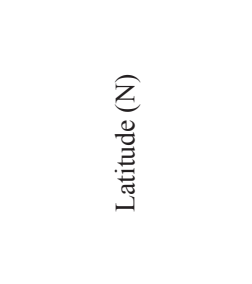 } & 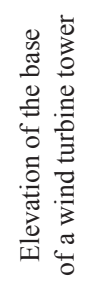 & 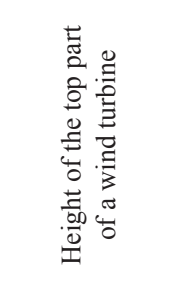 & 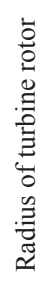 & 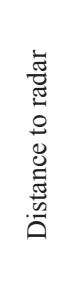 & 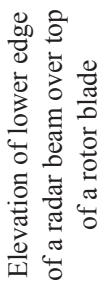 & 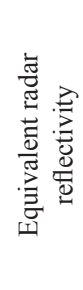 & 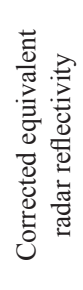 \\
\hline & \multicolumn{3}{|c|}{$\lambda_{W T}$} & \multicolumn{3}{|c|}{$\varphi_{W T}$} & $H_{W T}$ & $h_{W T}=h_{w}+R_{r o t}$ & $R_{\text {rot }}$ & $d$ & $W z n_{w}$ & $Z$ & $Z_{c o r}$ \\
\hline & 。 & , & " & $\circ$ & , & $"$ & $\begin{array}{c}{[\mathrm{m}]} \\
\text { AMSL }\end{array}$ & $\begin{array}{l}{[\mathrm{m}]} \\
\text { AGL }\end{array}$ & [m] & {$[\mathrm{km}]$} & {$[\mathrm{m}]$} & [dBZ] & [dBZ] \\
\hline Dębinki I & 20 & 43 & 16,5 & 52 & 34 & 7,03 & 97 & 150 & 45 & 24,3 & $-94,2$ & 71,9 & 67,2 \\
\hline Dębinki II & 20 & 42 & 38,7 & 52 & 32 & 44,8 & 97 & 150 & 45 & 23,1 & $-97,8$ & 72,4 & 67,7 \\
\hline Dębinki III & 20 & 42 & 59,4 & 52 & 32 & 57,19 & 97 & 150 & 45 & 23,0 & $-97,9$ & 72,5 & 67,7 \\
\hline Dębinki IV & 20 & 43 & 3,34 & 52 & 32 & 43,9 & 97 & 150 & 45 & 22,7 & $-98,8$ & 72,6 & 67,8 \\
\hline Dębinki V & 20 & 42 & 29,6 & 52 & 32 & 56,61 & 97 & 150 & 45 & 23,4 & $-96,8$ & 72,3 & 67,5 \\
\hline
\end{tabular}

\section{Conclusions}

The growing demand for the installation of new wind turbines and the dwindling territory for their installation entails an increasing number of attempts to locate them in the vicinity of radar stations. To reduce time in the authorisation process of such investments, a simple but realistic tool is necessary. Some attempts have been made in this field. One of them is proposed in this article as a simplified model for the calculation of maximum potential clutters (as effective radar reflectivity in dBZ) caused by wind turbines. Based on the assumption that only moving, metallic parts of turbines cause echoes indelible by standard Doppler filtering, a method of preliminary evaluation of such clutter was developed, described in detail, illustrated with real examples, and finally discussed in this article.

An extended discussion of the results of the theoretical calculations for Dębinki wind farm (mentioned in the previous chapter) based on the model described in this article, as compared with real operational data from Legionowo radar station, based on two years' statistics and a set of examples, will be reported in the next article.

Bibliography

Angulo I., Grande O., Jenn D., Guerra D., de la Vega D., 2015, Estimating reflectivity values from wind turbines for analyzing the potential impact on weather radar services, Atmospheric Measurements Techniques, 8, 2183-2193, DOI: 10.5194/amt-8-2183-2015

Bobillot G., Rasoanaivo L., Nguyen P., Chaumette E., Fargette P., Petex J.-F., Langlet S., 2012, SiPRÉ, a software simulator of the perturbation of radars by wind turbines, [in:] Proceedings of ERAD 2012, Toulouse, 24-29 June, available at
http://www.meteo.fr/cic/meetings/2012/ERAD/extended_ abs/SP_042_ext_abs.pdf (data access 23.02.2017)

Cameron A., 2011, "Stealth Blades" Get Turbines Under the Radar, Renewable Energy World, News, 23 November, available at: http://www.renewableenergyworld.com/articles/print/special-supplement-wind-technology/volume-1/issue-5/windpower/stealth-blades-get-turbines-under-the-radar.html (data access 23.02.2017)

Crispin Jr. J.W., 2013, Methods of radar cross-section analysis, Elsevier, $440 \mathrm{pp}$.

Danoon L., El-Makadema A., Brown A., 2014, On the integration of lightning protection with stealth coated wind turbine blades, Wind Energy, 17 (10), 1577-1585; DOI: 10.1002/ we. 1652

Doviak R.J., Zrnič D.S., 2006, Doppler radar and weather observations, $2^{\text {nd }}$ edition, Dover Books on Engineering, Dover Publications, $562 \mathrm{pp}$.

Greving G., Malkomes M., 2006, On the concept of the radar cross section RCS of distorting objects like wind turbines for the weather radar, [in:] Proceedings of ERAD 2006, available at https://www.semanticscholar.org/paper/On-theConcept-of-the-Radar-Cross-Section-RCS-of-Greving-Malk omes/9cbb9aea3411ae344b90e634ac9d124d90de9c41 (data access 23.02.2017)

Meeus J., 1999, Astronomical algorithms, $2^{\text {nd }}$ edition,WillmanBell Inc., 477 pp.

Met Office, 2012, Safeguarding our observations. Met Office guidelines for wind farm developers: meteorological radar and other technical sites used for meteorology, available at: http://www.metoffice.gov.uk/media/pdf/9/b/Safeguarding_ our_Observations_Met_Office_guidelines_for_wind_farm _ developers_April_2012_v1.pdf (data access 23.02.2017)

NOAA, 2015, How the ROC analyses wind turbine siting proposals, NOAA's National Weather Service, Radar Operation- 
al Center, available at http://www.roc.noaa.gov/WSR88D/ WindFarm/Analyses.aspx?wid=dev (data access 23.02.2017) OPERA, 2010, Statement of the OPERA group on the cohabitation between weather radars and wind turbines, Deliverable OPERA_2010_14

SELEX ES, 2015, GDRX® Signal Processing. User Manual, Document Release 4.2 manual by SELEX ES GmbH, GEMA-SD-0104-0116-0042

CSZH, 2014, Electromagnetic performance analysis report (CSZH-C-6.5), Shanghai Zhihe FRP Co., Ltd.; CSZH Serial No. 1412
DoD, 2006, The effect of windmill farms on military readiness, Report to the Congressional Defense Committees, US Department of Defense, USA, 62 pp.

WMO, 2010, Commission for Instruments and Methods of Observation. Fifteenth session, Annex VI, WMO-No. 1064, available at https://www.wmo.int/pages/prog/www/CIMO/ CIMO15-WMO1064/1064_en.pdf (data access 23.02.2017) 